\title{
Semiconductor Laser Linewidth Measurements for Space Interferometry Applications
}

\author{
D. J. Dougherty, R. C. Guttierrez, S. Dubovitsky, and S. Forouhar \\ Jet Propulsion Laboratory MS302-306, \\ California Institute of Technology \\ Pasadena, CA 91109
}

\section{ABSTRACT}

\begin{abstract}
Narrow linewidth $(<100 \mathrm{KHz})$ semiconductor lasers are expected to be a key technology in NASA's stellar interferometry missions to search for planets around nearby stars. Long coherence length lasers are needed for precise (20 pm to $5 \mathrm{~nm}$ ) measurements of the optical path difference.

This work discusses results using the self-heterodyne delay technique to measure $1.3 \mathrm{um} \mathrm{InP} \mathrm{based} \mathrm{DFB} \mathrm{lasers.} \mathrm{We} \mathrm{will} \mathrm{also}$ address practical issues concerning detection and elimination of back reflections, choice of fiber length and resolution, and measurement of laser $1 / \mathrm{f}$ and current supply noise.
\end{abstract}

Keywords: DFB laser, delayed self-heterodyne interferometer, laser linewidth

\section{METROLOGY SOURCES FOR SPACE INTERFEROMETRY}

NASA's Deep Space 3 (DS3) mission will fly an array of spacecraft in formation to validate control and positioning technologies to be used in future orbiting stellar interferometers. A major goal is to search for terrestrial planetary systems around nearby stars. Interferometry allows an array of small aperture telescopes to achieve an angular resolution equal to that of an aperture with a diameter equal to their baseline separation. The angular resolution target for DS3 is 5 to 0.5 millaresec ( 5 to $0.5 \times 10^{-9}$ rad At $1 \mu \mathrm{m}$ wavelength, the diffraction limit implies a baseline separation of $1 \mathrm{~km}$ for the collecting apertures.

For this resolution to be achieved, distance metrology on the order of $5 \mathrm{~nm}$ to $20 \mathrm{pm}$ is needed to control the optical path difference of the collected starlight beams and to maintain telescope pointing accuracy. Optical interferometry using a network of metrology lasers will be employed to measure these small positional shifts. Semiconductor DFB lasers are very attractive for this application because they are compact, highly efficient, and mechanically rugged. The source must have a narrow spectral linewidth with a long coherence lengths are required for stable fringe formation over $\mathrm{km}$ range path length differences. A rough estimate of the necessary frequency stability based on practical measurement integration times is that the power spectral density (PSD) of the optical frequency fluctuation not exceed $455 \mathrm{~Hz} / \mathrm{Hz}$ in a bandwidth from $10 \mathrm{~Hz}$ to 1 kHz. For a white (spontaneous emission) limited laser, this corresponds to a Lorentzian linewidth FWHM of $100 \mathrm{kHz}$. Several groups have demonstrated semiconductor lasers with linewidths near or below $100 \mathrm{kHz}$. However, at low frequencies. semiconductor lasers are known to suffer from significant $1 / \mathrm{f}$ noise due to factors both internal and external to the laser, itself. Some form of electrical feedback will be necessary to counter-act slow frequency drifting.

Messurement of the frequency noise is necessary in order to design and test the stabilized laser. PSD measurements are normally conducted using a Fabry-Perot cavity to convert frequency excursions to intensity fluctuations which can be detected and displayed on a low frequency spectrum analyzer. For narrow linewidth lasers, high $Q$ cavities and detector with both high sensitivity high speed are required. Calibration is also a issue since the slope of the transmission with frequency must be accurately known. The self-heterodyne technique is straightforward to implement, is inherently calibrated, and gives a more direct picture of the laser spectrum. It is complementary to the Fabry-Perot PSD technique in that it responds primarily to high frequency phase noise. By measuring linewidths as a function of fiber delay, it is possible to estimate the contribution of excess frequency noise. Results reported in this work for two $1.3 \mu \mathrm{m}$ DFB lasers show that the PSD does have a frequency dependent contribution in addition to the white noise. but that the spectrum is not described well by the usually assumed $\mathrm{I} / \mathrm{f}$ spectrum. Efforts taken to identify and reduce crrors due back-reflections and current supply noise will also be 
discussed. Note, depending on the dominant spectral dependence of the frequency PSD, different conversions must be made to extract the optical spectral linewidth from the observed photo-current power spectrum (i.e. $1 / 2$ for white noise. $\sqrt{2} / 2$ for $1 / f$ noise). For this reason 'linewidth' will refer to the photo-current power spectrum FWHM unless other wise specified.

\section{SELF-HETERODYNE SYSTEM}

Self-heterodyne systems are described in many standard references $[5,6]$. For reference, the system used in this work will be briefly described. Two optical isolators are used at the entrance of the system to reduce back scatter from the optical fiber into the laser under test. On has a fixed $-60 \mathrm{~dB}$ return loss and the other is tunable with a $-40 \mathrm{~dB}$ return loss. Even with these precautions, back reflections were still a problem as will be discussed below. Next, the input beam is split using an 80 $\mathrm{MHz}$ acousto-optic frequency shifter. The beams are then coupled into the two arms of a $50 / 50$ fiber coupler, one of which is extended by a delay fiber of $5 \mathrm{~km}$ to $40 \mathrm{~km}$. Fusion splicing as well as FC-APC connectors were used to connect the fiber spools. The output beams from the coupler were collimated and sent to a high sensitivity detector for alignment, and a high speed detector and RF spectrum analyzer for spectral measurement.

Collimation of the light from the semiconductor chips was found to be the most crucial part of the system. Both conventional single-element AR coated aspheric objectives and lensed fiber tips were used. Serious problems due to back reflections were encountered using lenses. Chopping the beam and measuring the voltage drop across the laser with a lock-in proved that back scatter was present. This technique was also used to optimally set the tunable isolator. The observed beat spectrum showed large uncontrolled fluctuations an momentary side lobes at large frequency detunings indicating rapid wavelength shifting. Under video averaging, the spectrum was observed to 'breathe', slowy broadening and narrowing making linewidth determination impossible. Tapping on the input alignment mirror, before the isolators, caused large spectral disturbances. The same behavior was observed going directly into the isolators. This suggested that the problem was back-scatter from the diffuse light surrounding the collimated beam. Attempts at using additional AR coated lenses to focus the beams through the isolator's apertures did not help. The only remedy was to use tilted, clean ND filters before the isolators to attenuate the back scatter. With this precaution, accurate results could be obtained, but confidence could only be obtained through repeating measurements many times.

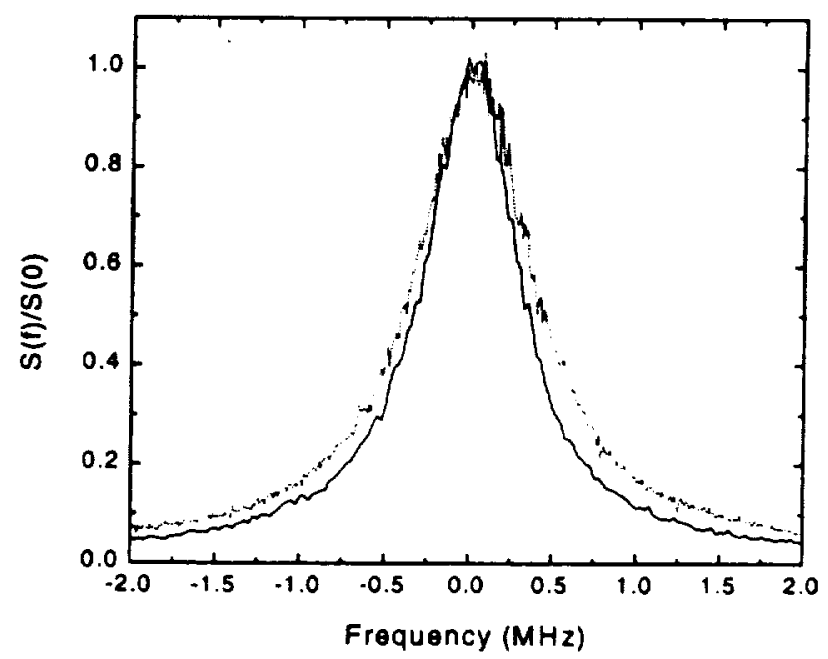

Figure 1 :Variation in spectral linewidth due to back reflections from a lensed fiber tip. 
Back-reflections were also observed using a lensed fiber. The lensed tip was spliced to a $-60 \mathrm{~dB}$ two stage fiber isolator and then to a low $(-60 \mathrm{~dB})$ fiber collimator. Up to $40 \%$ out coupling could easily be obtained, however, the same spectral 'breathing' was observed. Figure 2 shows repeated measurements, normalized to show changes in the FWHM, using a lensed fiber By backing the fiber off to $-2 \%$ out-coupling, the beat note spectrum became very stable and repeatable. At low powers the coupling could be increased, but usually was usually not adjusted to avoid possible errors in the power dependent measurements. The source of the back reflection has not been identified. By attaching a piezo to the fiber and periodically stretching it while measuring the laser voltage (or better current with a transformer) it should be possible to determine whether the source is the isolator, the collimator, or the tip itself. This is important because package lasers may employ fiber pigtailing with lensed fibers. Possible impact on the linewidth and spectral stability must be accounted for.

Recnt results by Prosyck et. Al. [7], have drawn attention to the role of current supply noise in limiting the observed linewidth. A standard, commerical battery-powered current supply (ILX model 3620) was used to drive the lasers. As in Ref [7], use of a $60 \mathrm{~Hz}$ line filter was also necessary to achieve minimum linewdith. Figure 2 shows the power dependent FWHM of the photo-current power spectrum for a $1.3 \mu \mathrm{m}$ DFB laser in different drive configurations. Without the filter (diamond symbols), but with the battery supply on, the linewidth levels out at $2 \mathrm{MHz}$. The solid circles are for the case of using the supply in AC mode but with the filter. The minimum linewidth in this case is about $715 \mathrm{kHz}$. Filtering can be more important than battery operation for low noise. The empty squares are for use of both the battery and filter and a minimum linewidth of $450 \mathrm{kHz}$ is observed.

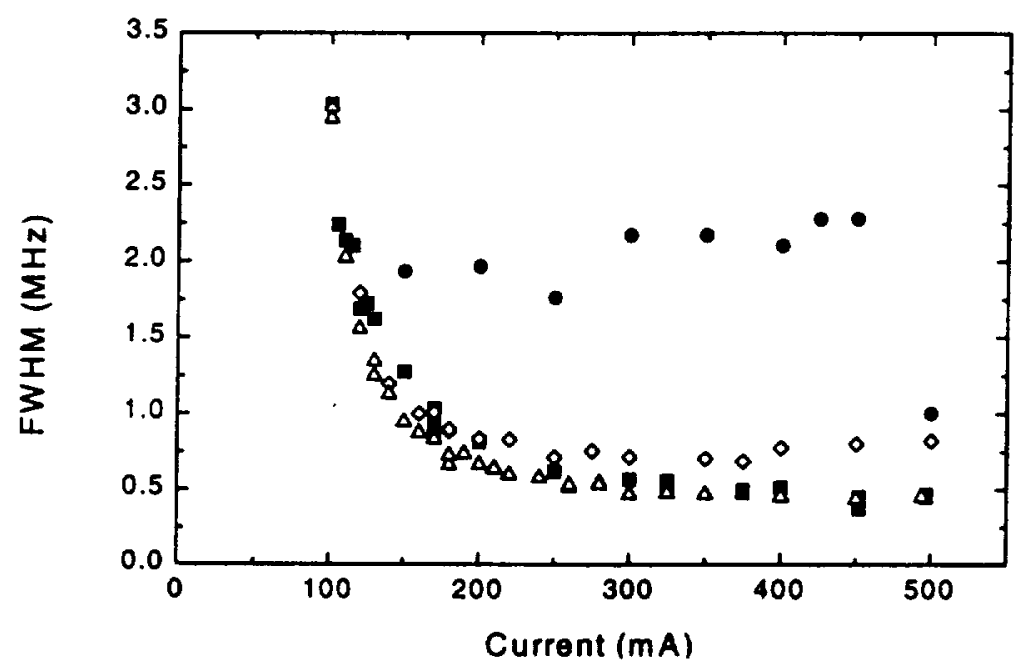

Figure 2 :Current dependence of spectral linewidth under different drive conditions. Solid Circles: battery supply with no line filter. Empty Diamonds: AC supply with line filter. Empty triangles: battery supply with line filter. Solid Squares: battery, filter. aspheric lens collimation.

When using a battery supply, it is necessary to ensure proper grounding to avoid pick-up of line noise. Figure 3 shows spectra of the same laser as in Fig. 2 at $350 \mathrm{~mA}$ drive current in battery mode with the line filter in. The dashed curve was taken with the chassis unplugged. The side lobes at $3 \mathrm{MHz}$ are presumably due to line noise pick-up in the drive circuit. The measured current tuning coefficient for this laser was $0.59 \mathrm{MHz} / \mu \mathrm{A}$. A current fuctuation of only $5 \mu \mathrm{A}$ can be responsible. The solid curve results from simply plugging the $A C$ cord in while remaining in battery mode. The side lobes are reduced by 


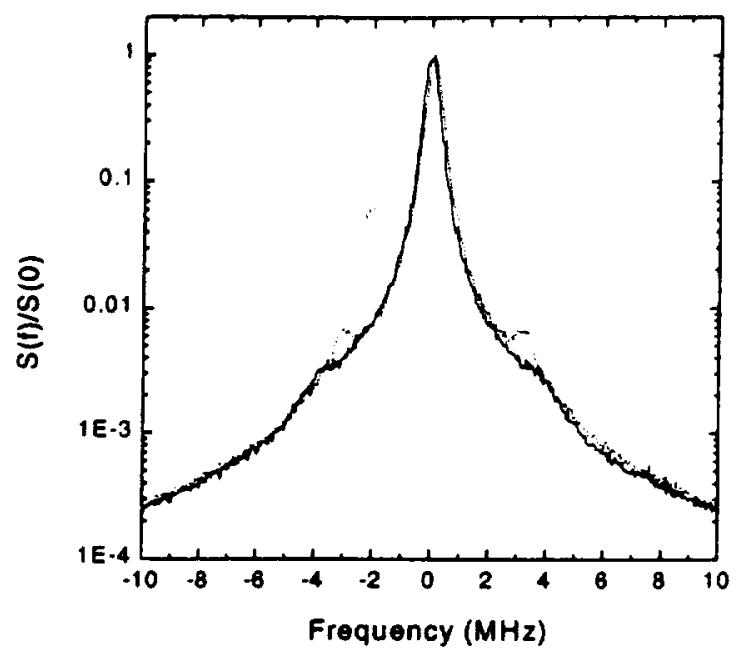

Figure 3: Effect of improper grounding under battery operation. Dotted curve: Supply chassis ungrounded, Solid curve: Chassis grounded with AC cord.

over $3 \mathrm{~dB}$ but are still evident. Further filtering and shielding of the entire laser and fiber mount in a steel box seems necessary to completely eliminate line noise.

\section{LINEWIDTH MEASUREMENTS}

With the above precautions taken, a number of $1.3 \mu \mathrm{m}$ DFB lasers were obtained for characterization. The lasers are ridge waveguides with holographic gratings, AR/HR facet coatings. They were mounted p-side down and have output powers of over $100 \mathrm{~mW}$. All measurements were performed with a TE cooler at $15 \mathrm{C}$. Results for two lasers at $7 \mathrm{~nm}$ and $20 \mathrm{~nm}$ detuning from the gain peak will be presented to compare the effects of minimizing the linewidth enhancement factor, $\alpha$ Figure 4 shows the power dependence of the electrical power spectrum FWHM of the lasers using a $5 \mathrm{~km}$ fiber. The left plot shows the linewidth of laser \#1, the $7 \mathrm{~nm}$ blue-shifted DFB. The minimum $3 \mathrm{~dB}$ linewidth is found to be $1.4 \mathrm{MHz}$ at $160 \mathrm{ma}$ $(47 \mathrm{~mW})$, . Above $200 \mathrm{~mA}(60 \mathrm{~mW})$ the linewidth suffers from the well-known effects of rebroadening due to spatial hole burning [4]. The right plot shows the results for the $20 \mathrm{~nm}$ blue-shifted DFB. The linewidth floor in this case is found to be $450 \mathrm{kHz}$. No re-broadening is evident up to the maximum current available from the current supply.
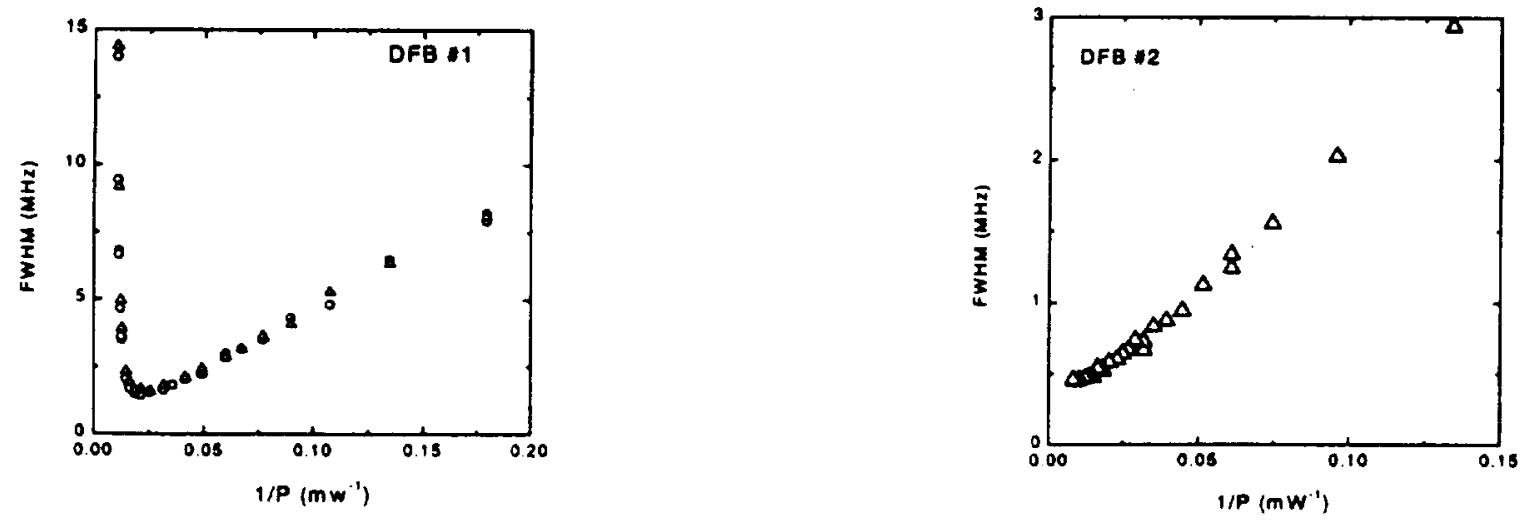

Figure 4: Linewidth power dependence for two DFB lasers. Left plot: DFB \#1, grating detuned $-7 \mathrm{~nm}$ from gain peak. Right plot: DFB \#2, grating detuned $-20 \mathrm{~nm}$ from gain peak. 


\section{FIBER LENGTH DEPENDENCE}

Attributing laser the linewidth of laser $\$ 2$ to spontaneous emission noise alone, an optical linewidth of around $225 \mathrm{kHz}$ can be estimated. This is encouraging since the implied PSD is only a factor of two from the frequency noise design goal and has been achieved with a simple device design. It is well known that estimation of the optical spectrum from -3db full widths of the electrical power spectrum can be inaccurate due to presence of $1 / f$ components in the frequency noise PSD. Procedures for examining at fitting self-heterodyne lineshapes with both white and $1 / \mathrm{f}$ noise are given in Ref [8]. Before discussing the measured lineshapes, results of changing the fiber length will be presented.

Equation $1[8,9]$ gives the self-heterodyne lineshape as a function of the laser frequency noise PSD

$$
S_{S H}(v)=\int_{-\infty}^{\infty} d \tau \exp (-i 2 \pi v \tau) \exp \left[-8 \int_{0}^{\infty} d f \frac{\sin ^{2}(\pi f \tau) \sin ^{2}\left(\pi f \tau_{d}\right)}{f^{2}} S_{P S D}(f)\right]
$$

where $\tau_{d}$ is the fiber delay time. The fiber interferometer acts as a high pass filter of the laser frequency noise. By changing the fiber length, information on the frequency noise spectrum for arbitrary PSD spectra can be obtained. It is usually assumed, however, that the frequency noise PSD has the form

$$
S_{P S D}(f)=S_{0}+\frac{S_{1}}{f}
$$

where $S_{0}$ is the white noise, inversely dependent on the optical power, and $S_{1}$ is a power independent $1 / f$ contribution. The $S_{0}$ term gives a term linear in $\tau$ in the argument of the exponential function in Eq. 1, and leads to a Lorentzian lineshape. Ref [9] provides an analytical expression for the $1 / \mathrm{f}$ contribution. Among other terms, a term quadratic in $\tau$ is added when $1 / \mathrm{f}$ noise is present. This leads to a Gaussian lineshape. The full self-heterodyne lineshape can thus be approximated by the convolution of a Gaussian and Lorentzian function. Since a Gaussian falls off much faster than a Lorentzian in the wings of the spectrum, the center of the measured lineshape will be most affected by $1 / f$ noise.

Figure 5 plots the measured FWHM at fixed optical power for different fiber lengths from 5 to $40 \mathrm{~km}$. Laser \#1 shows broadening from 1.4 to $1.6 \mathrm{MHz}$. Laser $\# 2$ shows on broadening from 550 to $730 \mathrm{kHz}$.. The solid lines are fits using Eq. 1 with $S_{0}$ and $S_{1}$ as parameters. The dependence of the lineshape FWHM on fiber length using Eq 1 was found to be quite gradual. To fit the observed length dependence, in both cases, the $1 / \mathrm{f}$ component was required to dominate over the white noise.
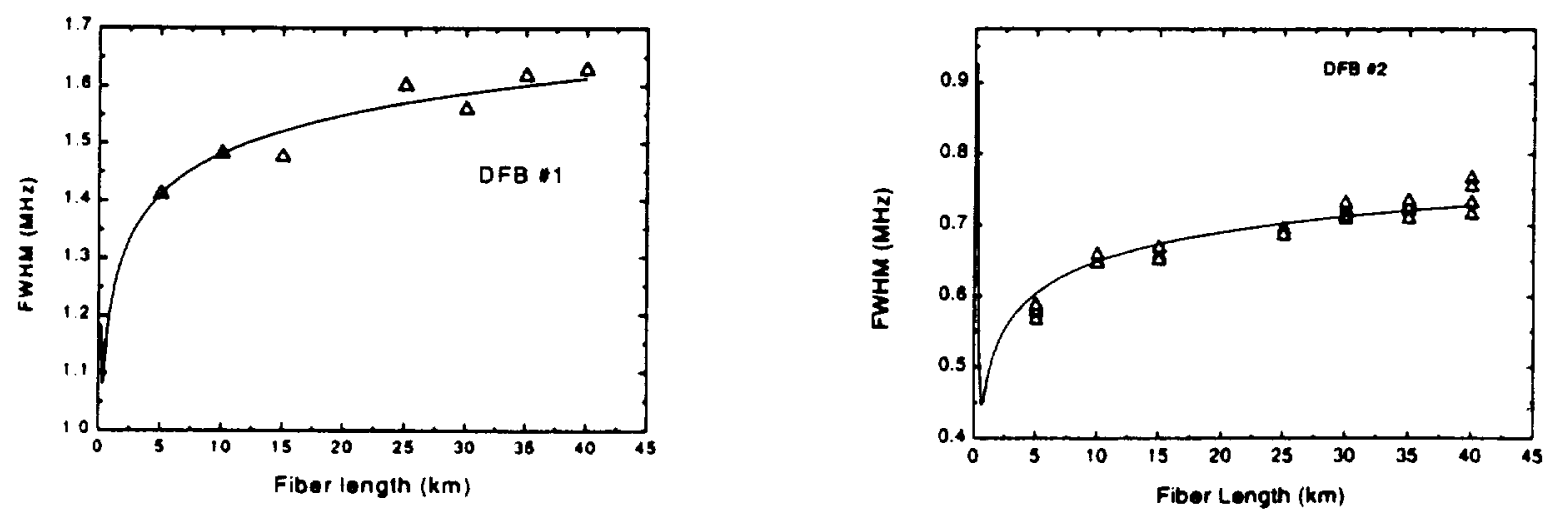

Figure 5: Dependence of FWHM on Fiber length 
For laser \#I, $S_{0}=.0875 \mathrm{MHz}$ and $S_{1}=.02 \mathrm{MHz}^{2}$ corresponding to individual component linewidths of $550 \mathrm{kHz}$ and $1.27 \mathrm{MHz}$ respectively at $40 \mathrm{~km}$ delay. For laser $\# 2, S_{0}=0.0175 \mathrm{MHz}$ and $S_{1}=.006 \mathrm{MHz}^{2}$ corresponding to linewidths of $110 \mathrm{kHz}$ and $660 \mathrm{kHz}$ respectively at $40 \mathrm{~km}$ delay. For each laser, the $1 / \mathrm{f}$ noise must account for almost the entire linewidth to fit the measured broadening with length.

Calculated spectra based on these parameters do not correspond to the measured lineshapes. Figure 6 shows spectra for each laser taken at $40 \mathrm{~km}$. The left plot shows the spectrum for laser \#1 along with three trial fits. The dashed curve is a $1.6 \mathrm{MHz}$ FWHM Lorentzian. This fit matches the spectrum's FWHM, but over estimates the width in the wings. The dotted curve is the calculated self-heterodynes lineshape using Eq. 1 and the paramters $S_{0}$ and $S_{1}$ determined from the measured fiber length dependence (Fig. 5 , left plot). This fit again matches the $-3 \mathrm{~dB}$ width, but underestimates the width of the wings. The best fit in the wings is obtained with a $1.4 \mathrm{MHz}$ FWHM lorentzian (solid curve). This is the same FWHM observed with a $5 \mathrm{~km}$ fiber, indicating that this is the white noise limited linewidth.
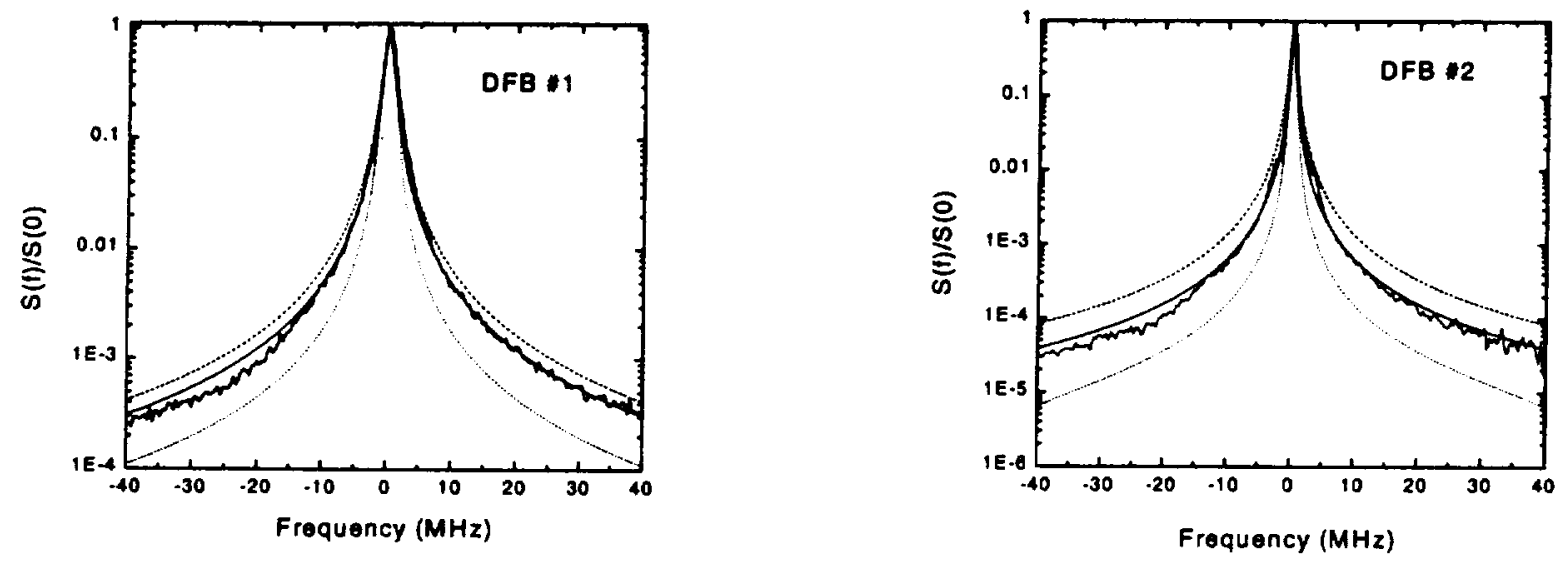

Figure 6 : Self-Heterodyne spectra with $40 \mathrm{~km}$ fiber. Left plot: Dashed fit: $1.6 \mathrm{MHz}$ Lorentzian. Dotted fit: calculated self-heterodyne lineshape (see text). Solid fit: $1.4 \mathrm{Mhz}$ Lorentzian. Right plot: Dashed fit: $730 \mathrm{kHz}$ Lorentzian. Dotted fit: calculated self-heterodyne lineshape (see text). Solid fit: $500 \mathrm{kHz}$ Lorentzian.

The right plot for laser \#2 shows similar behavior. The dashed curve is a lorentzian fit matching the FWHM of $760 \mathrm{kHz}$. The dotted curve is the calculated self-heterodyne lineshape width a white noise component of $110 \mathrm{kHz} F W H M$, and as with laser \#I, it still underestimates the wings. The solid curve is a $500 \mathrm{kHz}$ Lorentzian which, again, is comparable to the observed FWHM at $5 \mathrm{~km}$ delay. 
Table 1

\begin{tabular}{|l|l|l|l|l|l|l|}
\hline Laser \#l & & Exp. & Exp. & Calc & Calc & Calc \\
\hline & & $\Delta v_{\text {FWHM }}(-3 \mathrm{~dB})$ & $\Delta v_{\text {FWHM }}(-20 \mathrm{db})$ & $\Delta v_{\text {FWHM }}\left(S_{0}\right)$ & $\Delta v_{\text {FWHM }}\left(S_{1}\right)$ & $\Delta v_{\text {FWHM }}($ full $)$ \\
\hline & & & & & & \\
\hline & $5 \mathrm{~km}$ & $1.4 \mathrm{MHz}$ & $1.4 \mathrm{MHz}$ & $550 \mathrm{kHz}$ & $1.06 \mathrm{MHz}$ & $1.41 \mathrm{MHz}$ \\
\hline & $40 \mathrm{~km}$ & $1.6 \mathrm{MHz}$ & $1.4 \mathrm{MHz}$ & $550 \mathrm{kHz}$ & $1.28 \mathrm{MHz}$ & $1.61 \mathrm{MHz}$ \\
\hline & & & & & & \\
\hline Laser $\# 2$ & & & & & & \\
\hline & $5 \mathrm{~km}$ & $570 \mathrm{kHz}$ & $400 \mathrm{kHz}$ & $110 \mathrm{kHz}$ & $540 \mathrm{KHz}$ & $604 \mathrm{KHz}$ \\
\hline & $40 \mathrm{~km}$ & $730 \mathrm{kHz}$ & $500 \mathrm{kHz}$ & $110 \mathrm{kHz}$ & $660 \mathrm{KHz}$ & $730 \mathrm{KHz}$ \\
\hline & & & & & & \\
\hline
\end{tabular}

Table 1 is a summary of the observed and fitted linewidths for both lasers. The first two columns are the FWHM of Lorentzian fits to the measured spectra. In the first column, the fit is design to match the measured spectrum at the $-3 \mathrm{~dB}$ points. In the second column, the fit is to the wings, $-20 \mathrm{~dB}$ down. Thus, laser $\# 1$ shows the expected behavior: at short delays, white noise dominates and a Lorentzian fit is valid over the entire spectrum. At longer delays, extra frequency noise is integrated, and the FWHM is slightly broadened with respect to the wings. The last three columns refer to calculated fits to the data in Fig. 6 using the 1/f noise model in Eq. 1. Columns 3 and 4 are the FWHM of the white noise and 1/f noise spectral components, which are convolved to get the full lineshape. The last column shows the FWHM of the full calculated lineshape. (Note, column 5 is designed to match column 1, the measured FWHM.)

The data in first two columns suggest that the effects of excess frequency noise is small, and that the lasers (in particular laser \#1) are limited primarily by spontaneous emission noise. The calculated FWHM in the last three columns seem to indicate that the measuremnts are limited by $1 / \mathrm{f}$ noise, which may not be intrinsic to the devices. This is believed to be incorrect, and is the result of the assumption the frequency noise PSD is given by Eq. 2 . Taking the frequency dependence as $1 / \mathrm{f}^{2}$ would give a stronger length dependence to the observed FWHM.

\section{CONCLUSION}

Narrow linewidth semiconductor lasers offer significant advantages to the design of the proposed NASA Space Interferometry Mission. In order for semiconductor lasers to become the baseline technology for this mission, accurate knowledge of the linewidth and frequency noise spectrum is necessary in order to verify that DFB lasers can meet the design specification and be integrated into the metrology system. This work has illustrated several sources of measurement error and has demonstrated way in which they may be overcome. An experiment to to estimate the frequency noise spectrum using self-heterodyning with an adjustable delay was conducted. The measured broadening does not agree with a $1 / f$ frequency noise spectrum. A $1 / \mathrm{f}^{2}$ model may give improved agreement. More importantly, the ability to easily identify the presence of excess noise should aid in design of appropriate shielding and drive circuits which become increasingly important as linewidth decreases.

\section{ACKNOWLEDGEMENTS}

The work was performed by the Center for Space Microelectronic Technology at the Jet Propulsion Laboratory, California Institute of Technology under contract with the National Aeronautic and Space Administration. 


\section{REFERENCES}

1. Y. Inaba, M. Kito, J. Ohya, M. Ishino, Y. Matsui, "Gain-Coupled DFB Lasers with reduced Optical confinement for Narrow Spectral Linewidth", paper TuCl, 16 IEEE International Semiconductor Laser Conference, Nara, Japan, 1998.

2. A. Mathur, M. Ziari, and V. Agrawal, "High Power $1.3 \mu \mathrm{m}$ Single Mode Lasers", paper TuC2, $16^{\text {th }}$ IEEE International Semiconductor Laser Conference, Nara, Japan, 1998.

3. B. Chen, W. Wang, X. J. Wang, J. Y. Zhang, H. L. Zhu, and Z. Fan, "High T $1.3 \mu \mathrm{m}$ AlGaInAs/InP DFB Laser with Complex-Coupled Grating Growth by LP-MOCVD", paper TuCS, $16^{\text {th }}$ IEEE International Semiconductor Laser Conference, Nara, Japan, 1998.

4. M. Okai, "Spectral characteristics of DFB semiconductor lasers and their improvements by corrugation-pitch-modulated structure", J. Appl. Phys 75 (1), I Jan. (1994).

5. L. E. Richter, H. I. Mandelberg, M. S. Kruger, and P. A. McGrath, "Linewidth determination from Self-Heterodyne Measurements with Subcoherence Delay times", IEEE J. Quantum . Electron., vol QE-22, pp.2070-2074 (1986).

6. A. Yariv, Optical Electronics, New York: Holt, Reinhardt, and Winston, 1985.

7. J. Goodwin, K. Prosyk M. Poirier, "Linewidth artifacts using the delayed self-homodyne technique", IEEE Photonics Technol. Lett., vol. , p. 1997.

8. L. Mercer, “1/f Frequency Effect on Self-Heterodyne Linewidth Measurements”, J. Lightwave Technol., vol. 9, p. 485 (1991).

9. K. Kikuchi, "Effect of 1/f type FM noise on semiconductor laser linewidth residual in the high power limit", IEEE J. Quantum Electron., col. QE-25,p. 6841989. 